\title{
Article \\ Smart Health-Enhanced Early Mobilisation in Intensive Care Units
}

\author{
Maria Ferre (D), Edgar Batista (D), Agusti Solanas (D) and Antoni Martínez-Ballesté *(D) \\ Department of Computer Engineering and Mathematics, Universitat Rovira i Virgili, \\ E43007 Tarragona, Catalonia, Spain; maria.ferre@urv.cat (M.F.); edgar.batista@urv.cat (E.B.); \\ agusti.solanas@urv.cat (A.S.) \\ * Correspondence: antoni.martinez@urv.cat
}

Citation: Ferre, M.; Batista, E.; Solanas, A.; Martínez-Ballesté, A. Smart Health-Enhanced Early Mobilisation in Intensive Care Units. Sensors 2021, 21, 5408. https:/ / doi.org/10.3390/s21165408

Academic Editor: Antonio Suppa

Received: 11 July 2021

Accepted: 6 August 2021

Published: 10 August 2021

Publisher's Note: MDPI stays neutral with regard to jurisdictional claims in published maps and institutional affiliations.

Copyright: (c) 2021 by the authors. Licensee MDPI, Basel, Switzerland. This article is an open access article distributed under the terms and conditions of the Creative Commons Attribution (CC BY) license (https:// creativecommons.org/licenses/by/ $4.0 /)$.

\begin{abstract}
Critically ill patients that stay in Intensive Care Units (ICU) for long periods suffer from Post-Intensive Care Syndrome or ICU Acquired Weakness, whose effects can decrease patients' quality of life for years. To prevent such issues and aiming at shortening intensive care treatments, Early Mobilisation (EM) has been proposed as an encouraging technique: the literature includes numerous examples of the benefits of EM on the prevention of post-operative complications and adverse events. However, the appropriate application of EM programmes entails the use of scarce resources, both human and technical. Information and Communication Technologies can play a key role in reducing cost and improving the practice of EM. Although there is rich literature on EM practice and its potential benefits, there are some barriers that must be overcome, and technology, i.e., the use of sensors, robotics or information systems, can contribute to that end. This article reviews the literature and analyses on the use of technology in the area of EM, and moreover, it proposes a smart health-enhanced scenario.
\end{abstract}

Keywords: early mobilisation; context-aware computing; sensors; smart health

\section{Introduction}

Post-Intensive Care Syndrome (PICS), or Intensive Care Unit Acquired Weakness (ICU-AW), is described as the disability that remains in patients surviving a critical illness after a long period in the ICU. Common symptoms include impairments in the physical function (e.g., fatigue, decreased mobility, etc.) to psychological health issues (e.g., anxiety, sleep disorders, cognitive issues, etc.). PICS, which is strongly associated with prolonged mechanical ventilation and bed-restore deep sedation, has been reported to occur on average in at least a quarter of ICU survivors [1].

Aiming at preventing PICS, Early Mobilisation (EM) protocols have been designed and are being applied during the patients' stay in the ICU. Such protocols consist of routines of mobilisations and exercises: from passive mobilisation of limbs and postural changes for sedated patients to walking and reproducing basic everyday movements, including active mobilisation of limbs and even electrostimulation. Figure 1 depicts a general scenario in a healthcare facility where patients undergo EM protocols. Morris et al. [2] first proposed a four-level protocol for the progressive mobilisation according to the conscious or unconscious state of patients, their cardio respiratory stability and muscle strength in their limbs. Protocols typically begin with a safety screening: patients must meet all criteria in order to start the EM routines. First stages of protocols, for unconscious patients, are based on passive mobilisations and patient turns. Once patients are conscious, they are required to perform active resistance exercises and change to sitting position. Final stages of EM protocols include active transfers to chairs, ambulating in hallways and using ergometers. 


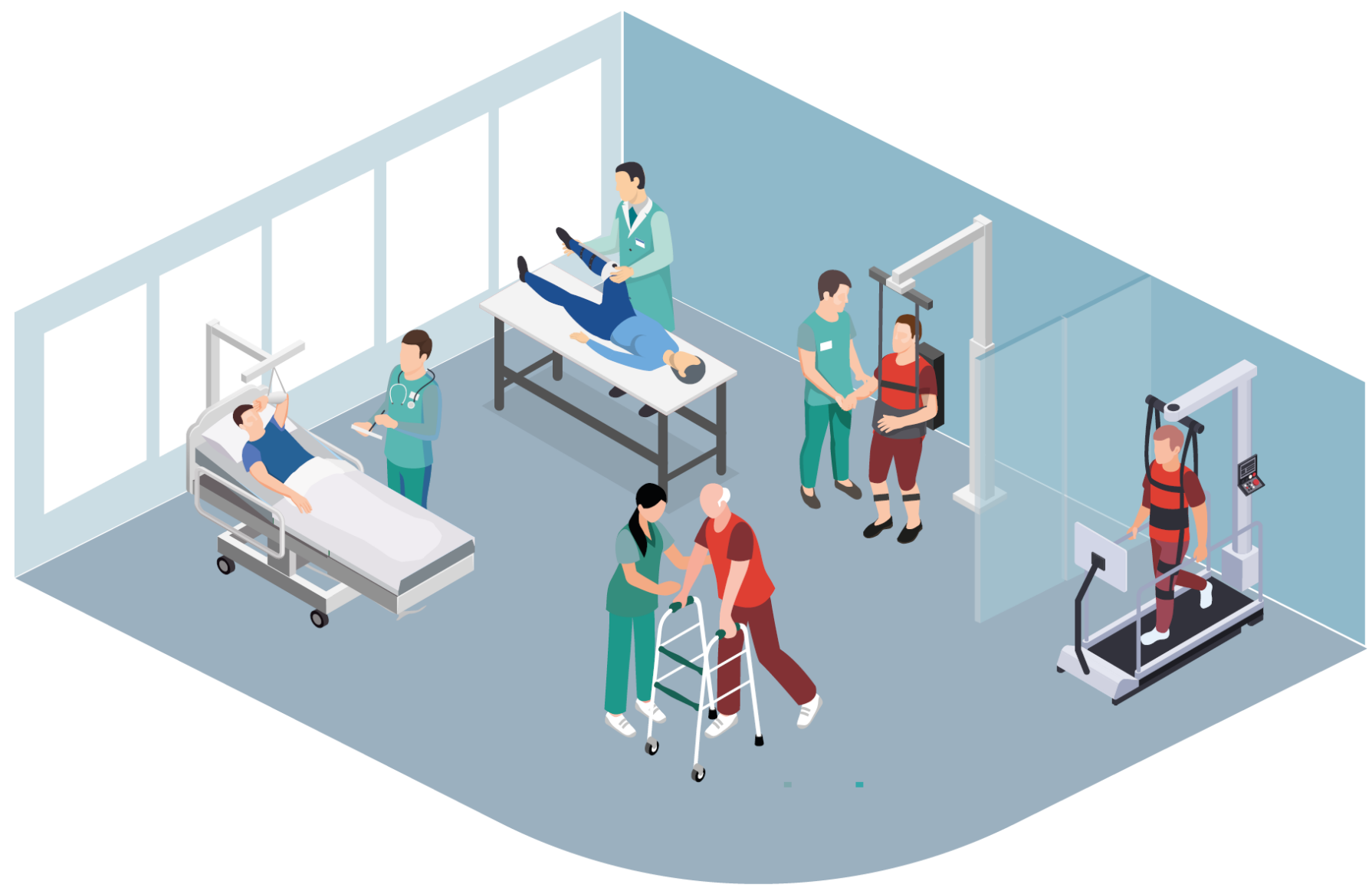

Figure 1. An Early Mobilisation scenario in a healthcare facility: patients in blue are undergoing passive mobilisations with the assistance of physical therapists (in green), and patients in red are performing active mobilisations.

A large body of literature is devoted to describing different approaches to EM, as well as to analyse their effects. A general consensus exists on EM being beneficial (i.e., patients have fewer ventilator-dependent days, shorter ICU and hospital stays, and better functional outcomes), and hence, it should be incorporated into daily clinical practice [3]. Last but not least, beyond generating substantial clinical improvements for ICU patients, EM reduces costs to hospitals [4]. However, the literature also considers several issues that require attention. Primarily, the wide variety of protocols and the different nature of ICU patients make it difficult for practitioners to adhere to a specific EM protocol. Furthermore, they prevent researchers from accurately studying EM effects and benefits. In this line, the optimisation of patients outcomes will require further studies on mobilisation timing and intensity, particularly within specific ICU populations [5]. Moreover, there are a number of elements that are perceived as barriers to the widespread implementation of EM, which are discussed next.

\subsection{Barriers to Early Mobilisation}

The literature on EM is essentially focused on the effects and potential benefits of such procedures: plenty of studies, reviews and surveys consider EM beneficial. Notwithstanding, a number of studies aim to identify the barriers to EM practice and its drawbacks. Aiming at identifying such issues, several studies use questionnaires $[6,7]$ and identify three levels of barriers [8,9]:

- Patient-level barriers: related to patient safety and efficacy of EM (e.g., medical instability, endotrach intubation, obesity, cognitive impairment).

- Institutional-level barriers: such as a lack of equipment or unclear guidelines.

- Provider-level barriers: such as limited staff (issues typically reported by physical therapists), problems in communication and protocol continuity over shift changes. 
Regarding the latter, experts suggest focusing on strategies such as increasing staffing and purchasing EM equipment in order to run successful EM programmes [9]. The sustainability of EM protocols has been described as essential for the successful implementation of EM, along with multidisciplinary team collaboration, data collection and feedback systems. Unfortunately, the intense daily activities in the ICU make fluid communication and appropriate data annotation puzzling. Nurses, physicians and physical therapists must proceed with EM protocols described on a document and must face monitoring with a scarcity of resources. All in all, communication between practitioners is essential to conduct safe and effective EM [10]. Moreover, the engagement of practitioners, patients and family is also considered a key component for EM practice, to which better communication could contribute [11].

\subsection{The Evolution of Healthcare Paradigms}

Information and Communication Technologies (ICT) have attracted the attention of the healthcare community: biometric sensors, robotics, electronic health records, etc., are concepts adopted in the everyday practice of medicine and healthcare. Electronic healthcare (or e-health), that is, "health services and information delivered or enhanced through the Internet and related technologies" [12], has become an essential cornerstone in medicine. Ehealth enables the provision of online medical treatments, the exchange of electronic health records in a standardised way between healthcare providers, and remote communications between patients and practitioners [13]. More recently, the advent of smartphones has resulted in their acceptance as important tools in the healthcare practice. In this line, mobile healthcare (or m-health), which could be defined as the use of "emerging mobile communications and network technologies for healthcare systems" [14], helps improve the communication between patients and practitioners, enables remote- and self-monitoring and defines medicine in a highly personalised, patient-centric perspective $[15,16]$.

Along with the abiding implementation of ICT in the healthcare domain, cities started to equip their infrastructures with ICT to face both urbanisation challenges, such as the increase of population moving from rural areas to urban areas, and demographic challenges, such as the world's population increase in life expectancy. ICT build up cities with sensing and analysis capabilities able to gather and exploit data in real-time to help decision-makers rearrange resources more efficiently and enhance the quality of life of citizens. Consequently, cities are steadily being transformed into smart cities, wherein citizens can interact with their immediate environment. By generalising this concept, environments capable of adapting themselves to users needs are commonly known as context-aware environments. Smart homes, smart buildings, smart hospitals and smart cities are examples of context-aware environments. The natural synergy between e-health and $\mathrm{m}$-health converged with the emergence of smart cities and context-aware environments, and inspired smart healthcare (or s-health), introduced in [17] as "the provision of health services by using the context-aware network and sensing infrastructure of smart cities". Although the provided definition was inspired within the scope of smart cities, the concept goes beyond their boundaries, and it could be generalised to context-aware environments. In addition to the patient-centric perspective used by both e-health and m-health paradigms, s-health also introduces an environmental/contextual approach, as it considers data coming from the sensing infrastructure of the context-aware environment and adapts its behaviour accordingly $[18,19]$. The generalised use of the Internet of Things (IoT) and low footprint sensors, which are able to gather vast amounts of contextual data with reduced computing capability and energy consumption, can contribute to the massive deployment of smart, context-aware healthcare solutions at large [20].

\subsection{Contribution and Plan of the Article}

This article discusses how technology, i.e., the use of sensors, information systems, biomedical electronic devices, etc., can contribute to the improvement of the practice of EM. Specifically, we aim to answer the following research questions: 
- Q1: Does the relevant literature on EM practice consider the use of technology?

- Q2: Does the literature on ICT include applications to EM?

- Q3: How can technology (e.g., e-health, sensors, robotics, etc.) help overcome barriers to EM practice?

To answer these questions, we have adopted the methodology proposed by Vom Brocke et al. for conducting literature reviews [21]. According to this methodology, we have addressed the definition of the review scope, conceptualised the topic and conducted the literature search. Section 2 describes these first phases, specially the identification of the relevant literature. Answering Q1 and Q2, in Section 3, we have analysed the selected literature by highlighting the aspects related to technology. Next, Section 4 answers Q3 through presenting a scenario for enhancing EM via technology and the smart healthcare paradigm. Finally, Section 5 provides some conclusions and final remarks.

\section{Methodology}

In this section, we describe the process of selecting the literature that will be analysed to address Q1 and Q2. Next, we define the review scope, discuss on the conceptualisation and describe the selection methodology.

\subsection{Definition of the Review Scope}

The focus of the review is to identify articles that are focused on the relationship between technology and EM, e.g., by considering technology as an important factor in EM practice or by presenting technological solutions aiming at improving EM. Our goal is to select and synthesise the literature on the topic so as to depict the use of technology in EM globally. We will organise results by grouping proposals and adopting a neutral but critical position. The review is intended for both practitioners in the healthcare area and professionals in the sector of ICT, which aim to comprehend how technology can contribute to EM success.

\subsection{Conceptualisation}

According to [22], 'early mobilisation' is the most frequently used term for the topic. However, this article clarifies that 'early rehabilitation' is often used in some articles, especially in Europe. Some other infrequent terms in the literature are 'early activity' or 'early physical therapy', among others. Furthermore, there is some literature that addresses the early mobilisation of extremities after injuries or surgeries. For the sake of clarity, we have concentrated on the concept of 'early mobilisation' or 'early rehabilitation' whenever they refer to protocols aiming at performing "a range of bodily movements carried out by care provider as part of care for a critically ill patients admitted into the ICU, which may include active or passive movement in bed, sitting up in bed, sitting by edge of bed, early transfer out of bed, sitting and standing out of bed, ambulation, ...", as described in [22]. Hence, articles addressing issues such as rehabilitation after injuries will be considered out of the scope.

Technology is a broad concept that encompasses a number of terms and disciplines: from simple mechanical equipment to the widely-spread ICT, namely computerised information systems, mobile applications, robots or sensors, to name a few. Since one of the identified barriers to EM is the appropriate monitoring and information acquisition on the practice of EM routines, the so-called Human Activity Recognition discipline (HAR) can play a key role. HAR is the automatised detection and understanding of actions performed by individuals [23], which can be applied in a number of different areas, e.g., ambient assisted living, surveillance, physical rehabilitation, etc. Its development is supported by software in the fields of computer science and machine learning and by sensors (cameras, accelerometers, etc.) as well. Another technology-related discipline that may contribute to EM practice is robotics. For instance, robots can help perform passive mobilisations of extremities. Furthermore, software can contribute to overcoming many of the barriers to EM practice, e.g., from applications aiming to ease the adherence to EM protocols to apply- 
ing data mining or process mining techniques. These aspects will be further addressed in Section 3.

\subsection{Literature Selection}

Our approach to literature selection is aimed at obtaining three sets of contributions:

- Set 1 . The most recent reviews and surveys on EM that consider/mention technology in their analyses or discussions. The articles in this set will be used to address Q1.

- Set 2. The most-cited contributions on EM that mention the use of technology. The articles in this set will also be used to address Q1.

- Set 3. Original research articles on the application of technology to EM. The articles in this set will be used to address Q2.

To this end, a two-phase review methodology, consisting of an initial search phase and a latter screening phase, has been followed. This methodology is depicted in Figure 2. First, the search phase aims to identify a preliminary list of potentially relevant works and, to this end, some queries were designed and executed on scientific literature databases. Regarding Set 1 , we searched the Web of Science (WoS) databases, specifically WoS-core collection and WoS-medline (the primer life sciences database), using the following search string:

$$
\begin{aligned}
& S 1=(\text { topic }=\{\text { "early mobili*ation" OR "early rehabilitation" }\}) \text { AND }(\text { title }=\{\text { "review" } \\
& \text { OR "survey" }\}) .
\end{aligned}
$$

The topic field includes title, abstract, author keywords and keywords plus (https:/ / support.clarivate.com/ScientificandAcademicResearch/s/article/KeyWords-Plus-generationcreation-and-changes, accessed on 1 May 2021). Wildcard * is used to query using both spellings of the same word (e.g., mobilisation, mobilization). Due to the amount of results (771 results) and aiming at focusing on recent surveys, we selected articles published in the last 5 years.

Regarding Set 2, we used the following search string:

$$
S 2=(\text { topic }=\{\text { "early mobili*ation" OR "early rehabilitation" }\})
$$

Note that the number of citations can be considered as a measure of the quality of a publication [24]. In order to select the most cited-works, we only considered articles with more than 100 citations according to WoS.

To obtain the preliminary candidates for Set 3 , which focuses on technology applied to $\mathrm{EM}$, we conducted searches in IEEE Xplore (which indexes documents from publications in ICT areas, e.g., computer science, telecommunications or electronics) and the ACM Digital Library (that provides access to documents in computing-related areas). We have used the following search string:

S3 $=($ fulltext\&metadata $=\{$ ("early mobili*ation" OR "early rehabilitation" $)$ AND ("HAR" OR "human activity recognition" OR "sensors" OR "robotics" OR "software" OR "artificial intelligence")\})

For Set 2 and 3, we did not apply any timespan criteria to our search. For Set 3, the search was done over the entire text and metadata. Searches were conducted on 31 May 2021. Duplicated or non-English articles were removed. Moreover, some of the articles were misidentified: eight original research articles were obtained for Set 1 and were not included into Set 2 because they had less than 100 citations; similarly, four surveys were removed from the results of Set 2 and could not be included in Set 1 because of their publication date.

In addition to these searches, we conducted a selection of articles using the MDPI database. Specifically, the following search string was used:

$$
S_{\text {add }}=(\text { all fields }=\{\text { "early mobili*ation" OR "early rehabilitation" }\})
$$


This search produced 238 results. In the identification phase, title and abstracts were revised in order to classify the articles as recent surveys, original research articles or articles on technology.

After the identification phase, two of the authors performed a blind screening, whereas other authors contributed to breaking ties: hence, if the two reviewers have different opinions, a third one participates in the final decision. For Set 1, full articles were screened (whenever the full paper was available) to assess to what extent they consider technology, i.e., the survey does not mention the use of technology, the survey highlights some uses of technology, or the survey considers technology in the analysis and classification of studies. For Set 2, titles and abstracts were reviewed to determine whether the articles fit with our concept of EM: for instance, articles addressing the mobilisation of joints after injury, articles addressing post-operative procedures and articles related to newborn ICUs were identified as out of the scope. Moreover, the screening phase filtered out articles not addressing technology. Finally, for Set 3, both titles and abstracts were screened. However, for Sets 2 and 3, the full-text of the paper was reviewed, if available, in case titles and abstracts did not provide enough information to classify the paper.

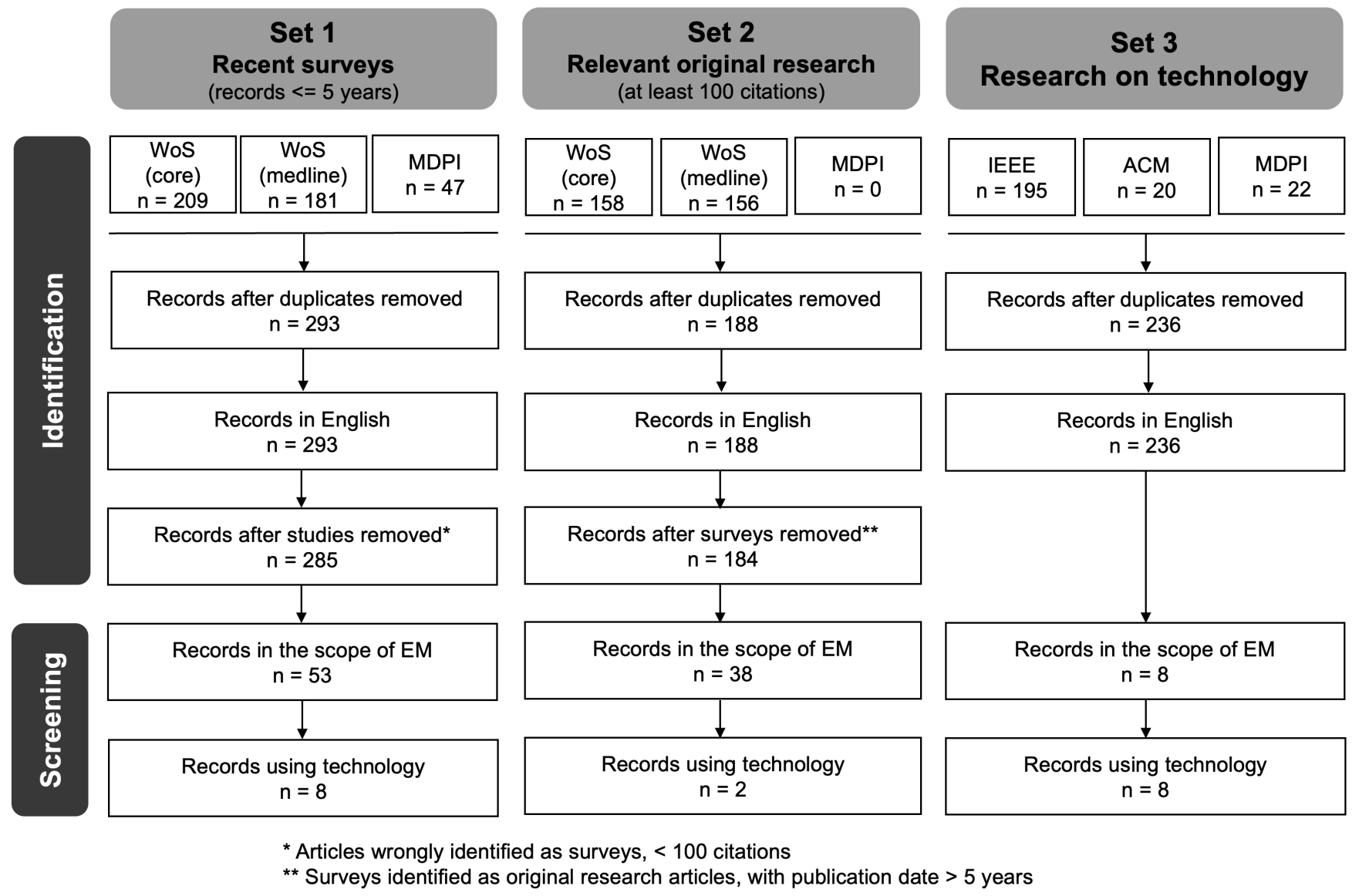

Figure 2. Synthesis of the search, screening and selection methodology.

\section{Results}

After the screening phase, Sets 1, 2 and 3 contained eight, two and eight articles, respectively, as shown in Table 1 . In this section, we overview the main findings after analysing these articles. 
Table 1. A summary of the results after the screening phase, with publication years and number of citations in May 2021. The results are classified according to the kind of technology they consider: neuromuscular electrical stimulation (NES), robotics and mechatronics and sensors. Papers $[25,26]$ approach technology from a global perspective.

\begin{tabular}{|c|c|c|c|c|c|c|}
\hline Set & Title & Year & Citations & NES & Robotics & Sensors \\
\hline \multirow{8}{*}{$\begin{array}{l}\text { Set } 1 . \text { Surveys on EM } \\
\text { mentioning technology }\end{array}$} & $\begin{array}{l}\text { Early mobilization and physical exercise in patients with } \\
\text { COVID-19: A narrative literature review [27] }\end{array}$ & 2021 & 0 & $\bullet$ & & $\bullet$ \\
\hline & $\begin{array}{l}\text { Rehabilitation to enable recovery from COVID-19: a rapid } \\
\text { systematic review [28] }\end{array}$ & 2021 & 0 & $\bullet$ & & \\
\hline & $\begin{array}{l}\text { Review: How Can Intelligent Robots and Smart Mechatronic } \\
\text { Modules Facilitate Remote Assessment, Assistance, and } \\
\text { Rehabilitation for Isolated Adults With Neuro-Musculoskeletal } \\
\text { Conditions? [29] }\end{array}$ & 2021 & 0 & & $\bullet$ & \\
\hline & $\begin{array}{l}\text { Three-Fourths of ICU Physical Therapists Report the Use of } \\
\text { Assistive Equipment and Technology in Practice: Results of an } \\
\text { International Survey [25] }\end{array}$ & 2021 & 0 & & & \\
\hline & $\begin{array}{l}\text { The effects of neuromuscular electrical stimulation in critically ill } \\
\text { patients: A systematic review and meta-analysis of randomised } \\
\text { controlled trials [30] }\end{array}$ & 2020 & 6 & $\bullet$ & & \\
\hline & $\begin{array}{l}\text { Gait rehabilitation after stroke: review of the evidence of } \\
\text { predictors, clinical outcomes and timing for interventions [31] }\end{array}$ & 2020 & 3 & & $\bullet$ & \\
\hline & $\begin{array}{l}\text { Actigraphy to Measure Physical Activity in the Intensive Care } \\
\text { Unit: A Systematic Review [32] }\end{array}$ & 2019 & 8 & & & $\bullet$ \\
\hline & $\begin{array}{l}\text { Physiotherapy in the neurotrauma intensive care unit: A scoping } \\
\text { review [33] }\end{array}$ & 2018 & 4 & • & & \\
\hline \multirow{2}{*}{$\begin{array}{l}\text { Set } 2 \text {. The most-cited } \\
\text { contributions on EM that } \\
\text { mention the use of } \\
\text { technology. }\end{array}$} & $\begin{array}{l}\text { An early rehabilitation intervention to enhance recovery during } \\
\text { hospital admission for an exacerbation of chronic respiratory } \\
\text { disease: Randomised controlled trial [34] }\end{array}$ & 2014 & 144 & $\bullet$ & & \\
\hline & $\begin{array}{l}\text { Technology to enhance physical rehabilitation of critically ill } \\
\text { patients [26] }\end{array}$ & 2009 & 127 & & & \\
\hline \multirow{8}{*}{$\begin{array}{l}\text { Set 3. Original research } \\
\text { articles on the application } \\
\text { of technology to EM }\end{array}$} & $\begin{array}{l}\text { The Effect of Multi-Frequency Whole-Body Vibration on Muscle } \\
\text { Activation, Metabolic Cost and Regional Tissue Oxygenation [35] }\end{array}$ & 2020 & 0 & & $\bullet$ & \\
\hline & $\begin{array}{l}\text { Joint Distribution and Transitions of Pain and Activity in } \\
\text { Critically Ill Patients [36] }\end{array}$ & 2020 & 0 & & & $\bullet$ \\
\hline & $\begin{array}{l}\text { e-PEMICU: an e-Health Platform to Support Early Mobilisation } \\
\text { in Intensive Care Units [37] }\end{array}$ & 2019 & 1 & & & $\bullet$ \\
\hline & $\begin{array}{l}\text { Postoperative healing patterns in elbow using electromyography: } \\
\text { Towards the development of a wearable mechatronic elbow } \\
\text { brace [38] }\end{array}$ & 2017 & 1 & & $\bullet$ & \\
\hline & $\begin{array}{l}\text { Real-Time Closed-Loop Control of Human Heart Rate and Blood } \\
\text { Pressure [39] }\end{array}$ & 2015 & 11 & & • & \\
\hline & $\begin{array}{l}\text { Novel tilt table with integrated robotic stepping mechanism: } \\
\text { design principles and clinical application [40] }\end{array}$ & 2005 & 14 & & • & \\
\hline & $\begin{array}{l}\text { Visualization of transfer motion based on accelerometry data in } \\
\text { the hemiplegic patients [41] }\end{array}$ & 2002 & 0 & & & • \\
\hline & $\begin{array}{l}\text { Evaluation of bed rest using a bed temperature monitor after } \\
\text { acute myocardial infarction [ } 42 \text { ] }\end{array}$ & 1995 & 1 & & & $\bullet$ \\
\hline
\end{tabular}

\subsection{Technology and Early Mobilisation}

One of the most cited works on EM addressing technology is [26], which has 127 citations. The article explores relevant technologies for rehabilitation of critically ill patients to prevent neuromuscular complications, namely neuromuscular electrical stimulation (NES), cycle ergometry and custom-designed technological aids and equipment for ambulation. The first two methods are more convenient at the early stages of the rehabilitation (even for sedated patients), whereas the latter requires the active participation of patients. As the latter is more challenging, authors presented a holistic system that improves the safety and effectiveness of ambulation of mechanically ventilated patients in the ICU, while reducing the number of human resources required to perform this task. Note that this article presents the outstanding technology that will be predominant in EM settings: on the 
one hand, passive EM using neuromuscular electrical stimulation, which creates passive contraction of muscles through the use of a low-voltage electricalised impulse delivered through electrodes; and on the other, active EM using cycle ergometers, i.e., stationary cycling apparatus with built-in mechanisms that can alter the exercise done by patients. Note that both cycle ergometers and electro-stimulators can to be programmed to perform a set of routines, with diverse intensities and lengths and, hence, can help practitioners apply precise and personalised EM protocols.

Physical therapists play a key role in the practice of EM. In order to asses the use of technology and equipment, ref. [25] presents the results of a survey completed by 206 practitioners. A total of $74 \%$ of respondents reported using at least one type of equipment or technology. Meanwhile, $31 \%$ of respondents reported using cycle ergometers, whereas the most common reason for not using equipment or technology was their limited or no availability. Approximately $75 \%$ of physical therapists emphasised the need for further research to better understand clinical approaches to the use and efficacy of technology.

\subsubsection{Neuromuscular Electrical Stimulation}

A number of articles consider NES as a pivotal element in EM protocols. In [34], which has 144 citations, authors concentrate on the use of NES in a trial where 196 patients underwent an EM intervention. Nevertheless, the approach of this article is slightly different since it concentrates on the outcomes instead of discussing the additional support of technology to EM. However, it is worth noticing that patients could continue with post-discharge training using electric stimulation at home. The surveys in $[30,33]$ relate 11 cases in which the effectiveness of NES is evaluated. In general, this technique is applied in 30-minute sessions once or twice a day. Last but not least, NES is also described as a successful and interesting practice in EM for patients with COVID-19 [27,28].

\subsubsection{Robotics and Mechatronics}

A robotic tilt table that allows early mobilisation of bed-ridden patients through modulating their body inclination and legs movement is presented in [39]. These parameters are automatically set by a self-learning fuzzy controller that is continuously monitoring and stabilising the cardiovascular parameters of patients, such as heart rate and blood pressure, to safe ranges. The proposed system aimed to effectively rehabilitate the deconditioned cardiovascular system following a long duration of bed rest in bed-ridden patients. Although the system was tested with healthy subjects, the cardiovascular parameters were successfully controlled within medically tolerable ranges during the realisation of early mobilisation exercises. The results were promising enough to study the suitability of the system in bed-ridden patients over prolonged periods. Similarly, ref. [40] proposed Erigo, a novel tilt table with an integrated robotic stepping mechanism for passive mobilisations. The proposed system aimed to recover the cardio-pulmonary system of patients with spinal cord injury or traumatic brain injury with passive movements in the patients' lower limbs. The customised verticalisation and stepping mechanism of the device enabled, for the first time, the generation of physiological load patterns. Although the results demonstrated a direct effect of the passive moment on the circulatory system, further studies with more patients and evaluating the long-term effects of the therapy are needed.

The authors in [35] proposed a pioneering wearable suit prototype aiming to provide whole-body mechanical vibrations, which are an effective treatment in rehabilitating immobilised patients to prevent muscle atrophy. Despite the benefits of whole-body vibration, there is a lack of technological solutions. This prototype was capable of delivering vibrations at multiple frequencies simultaneously to different parts of the body using inertial actuators. The results demonstrated an increase in muscle activation, tissue oxygenation and oxygen consumption. These increases were similar to those observed during mild physical activity, hence becoming a suitable solution to combat muscle weakness. However, these results were obtained from healthy patients, and its efficiency in critically ill hospitalised patients is still unknown. The authors in [38] developed a wearable mecha- 
tronic elbow trace to quantify the muscles health from electromyography (EMG) signals in musculoskeletal injured people. More specifically, six bipolar electrodes were placed over the patients' arms, and characteristics from EMG signals during the realisation of different exercises were extracted. By comparing the results between a group of healthy people and a group of people with a musculoskeletal injury or disorder in their elbows, the authors observed statistically significant differences in the magnitudes of muscle recruitment and activation between the two groups. Moreover, authors also noticed differences in the signals of injured patients according to their therapy stage: patients at the end of their therapies show similar trends closer to those of the healthy population. Hence, the healing progress changes the muscle activation patterns, which could ultimately lead to the improvement of therapy for rehabilitation robots.

Indeed, robotic-assisted therapy is increasingly used in the EM field, from walking exoskeletons to end-effector robots, including smart mechatronic wearable systems that aim at performing passive mobilisations $[29,31]$. They are appealing for EM as they can facilitate repetitive and intensive tasks training, provide assistance when needed, as well as deliver feedback. However, their high cost can be a barrier to their integration into EM protocols.

\subsubsection{Sensors to Monitor EM Routines}

Technology can also be used to improve the monitoring of EM routines. In this line, e-PEMICU is presented as an e-Health platform to support EM [37]. This solution, inspired in recent healthcare paradigms [17], is founded on the use of non-invasive motion sensors, smartphones, and further mobile devices to enhance ICUs in a cost-efficient and minimally intrusive manner. In addition to managing and recording EM sessions, practitioners can also objectively measure the degree of achievement of the performed routines as well as to properly analyse the collected data with data mining or process mining algorithms. The prototype was tested in a hospital environment, although no quantitative results were provided. In fact, the use of actigraphy is gaining the attention of professionals in the ICU and EM practitioners. According to [32], 16 studies describe the use of actimetry sensors (typically based on accelerometers) to measure patient activity in ICU settings.

Tsukamoto et al., in [41], describe the development of a quantitative monitoring device for rehabilitation training. The device, consisting of two tri-axial accelerometers attached to the patient's head and waist, enabled estimating the locus of patients' movement. By comparing healthy and hemiplegic patients, the authors observed differences in the height change at the head and at the waist, as well as the tremble in patients caused by the weakness of strength of the patients' legs. The main advantage of the suggested solution is that, as the patient's motion is depicted as graphics animations, therapists can easily understand the effectiveness of EM.

The proposal in [42] focuses on early rehabilitation of patients with acute myocardial infarction during their bed rest. Authors estimated the body movement of patients using several non-invasive temperature sensors and observed a positive correlation between an increased number of body movements with an increased heart rate. Hence, this measurement could be used to evaluate the physical activity for each recovery stage quantitatively.

Demrozi et al., in [36], studied the relationship between activity intensity and pain intensity in critically ill patients hospitalised in surgical ICUs. These measurements are essential indicators to assess the recovery progress of patients, maximise mobility and physical function, and reduce the prescription of opioids and other pain-relief medication. Unfortunately, authors detected a lack of studies examining this relation, and when conducted, measurements were manual and sporadic, limiting the extraction of actionable knowledge. On the one hand, activity intensity information was collected using three actigraphy devices, equipped with a tri-axial accelerometer sensor, placed on the wrist, arm and ankle. Furthermore, on the other hand, pain intensity was assessed hourly using the DVPRS scale (Defense and Veterans Pain Rating Scale, a scale that grades pain with a number, from 0 "No pain" to 10 "As bad as it could be, nothing else matters"), a well- 
known clinical practice. Although the results showed a relation between mild pain levels and high activity levels for the majority of the patients, some of them exhibited severe pain at low activity levels, indicating the need for optimising their early mobilisation routines.

Finally, the survey in [27], analyses 32 articles published in 2020 and focuses on several experiences that use telerehabilitation to deliver and monitor low-intensity EM programmes. Several proposals focused on COVID-19 patients and based on the use of tablets and motion sensors support that telerehabilitation is an important alternative to face-to-face exercises, without increasing the exposure and risk of contamination of practitioners [43-46].

\section{Discussion}

In this section, we first overview the limitations of the analysed articles. Moreover, we propose and discuss the concept of a smart healthcare-enhanced EM. Finally, we overview some existing proposals out of the scope of EM that could be integrated into our smart health-enhanced EM.

\subsection{Limitations of the Analysed Proposals}

According to our analysis, there is a reduced number of articles on EM that consider the use of electronic devices and ICT. Moreover, the existing proposals on solutions from the ICT area applied to EM merely address the use of a single technology (e.g., tilt tables, NES, etc.), but they do not present a holistic solution to improve EM practice. In this line, the proposals in the selected articles in Sets 1 and 3 describe technology-focused settings and the effects of using a specific kind of technology but, unfortunately, do not describe how the EM practise could be enhanced with techniques such as data mining, process mining or state-of-the-art machine learning. Only [37] suggests the use of artificial intelligence and the acquired data to assess the degree of achievement and adherence to the scheduled routines.

Notwithstanding, the reviewed technologies could be integrated into a global scenario for EM, enhanced via the adoption of the smart healthcare paradigm. Moreover, some of the proposals that were initially selected for Set 3 but were filtered out during the screening phase could be considered for this scenario.

\subsection{Smart Healthcare-Enhanced Early Mobilisation}

With the smart healthcare paradigm in mind, practitioners and patients could benefit from highly personalised programs, which consider patient physiological parameters as well as the context: the exercises conducted during EM programmes could be objectively monitored with a number of sensors attached to the patient's body, whose signals (e.g., collected from accelerometers) could verify whether the exercises are performed correctly. Besides, these sensors, data can be used to evaluate the evolution of patients and their adherence to the scheduled programmes, as well as to analyse the outcomes from a global perspective. Robotic-assisted therapy could be used to apply passive mobilisations. Additionally, the use of advanced data analysis techniques, such as artificial intelligence, data mining or process mining, may detect similarities or trends among patients, whose EM programmes could be slightly adjusted with personalised recommendations using the high-level knowledge acquired. The automatic real-time feedback received from sensors data could significantly shorten recovery times and minimise the PICS side-effects.

Figure 3 depicts a smart-healthcare-oriented scenario for EM. In this setting, physicians count with a system that considers input data (e.g., Electronic Health Records, physiological and context parameters acquired in real-time, activity data from actigraphs and motion sensors, etc.) to personalise the activities of the EM protocols. 


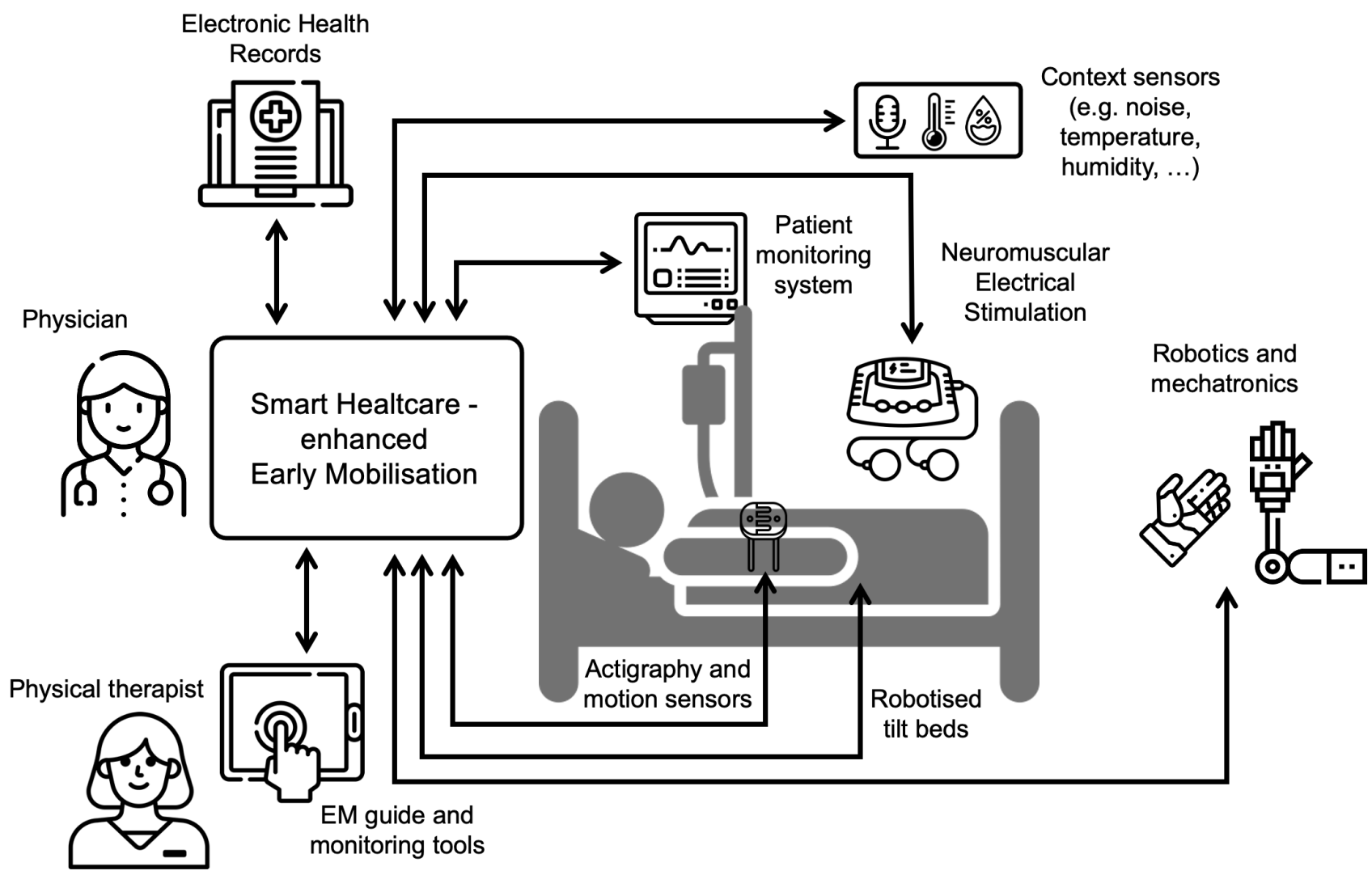

Figure 3. The components of a smart-healthcare-enhanced Early Mobilisation system.

Unfortunately, there are several shortcomings to overcome related to the connection and interoperability of these devices with information systems. One of them is the potential variety of devices involved in such systems. In this line, ref. [47] introduces an open-source middleware framework for communication with medical devices and an application using a middleware named Intensive Care Window. It enables communication with intensive care unit bedside-installed medical devices over standard and proprietary communication protocols. It facilitates the acquisition of vital signs and physiological parameters from medical devices and sensors. In addition, it provides tools for annotation, visualisation and analysis. Besides interoperability, issues in the physical layer must be taken into account: in such highly sensorised indoor settings, the coexistence of multiple wireless systems requires carefully considering potential interferences, strong multipath components and signal fading $[48,49]$. Another shortcoming is related to information security and privacy: devices and information systems store and transmit sensitive data (e.g., health parameters along with patient identifiers might be leaked upon attacks), and hence, several countermeasures must be considered, namely the use of authentication and encryption mechanisms for information security and pseudonymisation and other privacy enhancing technologies for data privacy [50-52].

To improve the application and outcomes of EM programmes, the design of a smarthealthcare-enhanced ICU and, especially, the conception of the patient room must consider the well-being and experience of patients, staff and visitors [53]. The design of the room should focus on functionality, and it needs to be capable of supporting the full scope of medical informatics (from devices to information systems). In addition to the emotional welfare of patients, the success of personalised EM programmes is also greatly impacted by the room's context. In this line, the acquisition of context data must be accompanied by a smart adaptation of the environment: sound-absorbent materials on the walls and ceiling, minimisation of sound alerts or usage of alternative vibration-based systems, natural 
lighting (or artificial ambient light systems to emulate day/night cycles), individual controls for temperature and humidity, etc., are essential in a successful smart healthcare scenario.

In our proposed solution, physical therapists and other users count with an electronic EM guide (in the illustration, operated using a tablet) with a schedule of mobilisations, and a monitoring tool to assess the precision and progression of the therapy. These software applications help overcome some of the provider-level barriers mentioned in Section 1.1 related to the management and communication between practitioners (i.e., team collaboration, data collection and feedback) as well as institutional-level barriers (e.g., unclear guidelines).

The EM guide could include video demonstrations of the mobilisations, so as to provide other caregivers and even patient relatives, with precise instructions on how to perform active mobilisations. As a result, a reduced group of physical therapists would be necessary to monitor and manage a growing number of personalised EM programmes without significantly having to increase resources. As a result, smart healthcare can help overcome the limited staff barrier to EM and, in the long term, promote its sustainability.

Besides physical therapists applying mobilisations by themselves, robotised elements may perform passive mobilisations to limbs, whereas NES can be applied at a specific pace and regulated according to patients' current vital sign status and response.

\subsection{Integrating Proposals from the Rehabilitation Area}

During the phase of literature identification, we discarded proposals that, although describing experiences based on technology, did not address EM programmes and practice. However, they are worth being considered for the integration into smart-healthcareenhanced EM scenarios.

Several of the literature articles that have not been selected are those that apply rehabilitation to patients who have suffered a stroke or patients with fractures or bone surgeries. Some of these articles present very specific rehabilitation equipment designs. For example, in [54], the design of a robot to rehabilitate after a knee operation is presented. In [55], a robotic sock (i.e., soft tissue) is used to rehabilitate the lower limbs and prevent thrombus due to lack of movement in patients who had a stroke. In [56], the use of an exoskeleton in the form of a glove for hand movements is described. The proposal in [57] combines the generation of electrical impulses to stimulate the exercises provided by an active robot on the patient's legs. Passive mobilisation using robotics is addressed in [58]. These kinds of proposals have a great impact on rehabilitation that is focused on a specific part of the body and could be easily integrated into smart-healthcare-enhanced EM.

EM protocols may not be only circumscribed within the ICU: once the patient is discharged from the ICU or the hospital, telerehabilitation could be used to continue with exercises and activities, whereas the progression of the physical and mental state of patients, as well as their adherence to the protocols, could be monitored. In [59], a home telerehabilitation system is presented. Sensor bands are placed on patients' arms, which capture the movements that are sent to a remote system for further analysis. Similarly, ref. [60] uses sensors to capture patients' movement in lower limbs exercises. In this case, the movement of the patient can be visualised in an application that provides real-time feedback while performing the exercise. Remote postoperative rehabilitation of lower limbs is addressed in [61], where a new smartphone application based on plantar pressure analysis is developed and tested.

Adherence to EM protocols can be improved through gamification: it encourages patients motivation, and hence, better loyalty in treatments is achieved [62]. Game-based rehabilitation is also explored in [63], where patients have to handle a cup and achieve milestones with it while controlling the movement they make with their arm. In [64], a set of sensors detect the movement of the feet, and this translates into the interaction in a game that works on a tablet. In [65], authors describe a virtual reality application that simulates a kitchen environment: patients use a robot to perform specific actions with their upper limbs, and these movements are transferred to the virtual kitchen. In the field of 
pulmonary rehabilitation, accelerometers have been used for monitoring physical activity and providing feedback that may increase patient motivation [66]. Finally, in [67], authors present a robotic design that allows stimulating the patient's gait despite being in bed and allows performing active and passive mobilisation. These articles, which focus on the patient motivation, show the technology needed to build these systems, and measuring their cost/benefit would be of interest, i.e., does the cost of building complex virtual reality systems improve rehabilitation performance accordingly?

\section{Conclusions}

The dependency on technology, ranging from sensors and electronic devices to advanced analytical techniques, is ever increasing in the healthcare industry. Care providers and healthcare organisations rely on emerging technology for improving patient care, shortening diagnosis and treatment times and decreasing healthcare expenditure in the long term. Besides, the emergence of novel healthcare paradigms based on context-awareness, such as smart healthcare, places technology as a pivotal actor in healthcare practice. Unfortunately, the role of technology in Early Mobilisation (EM) practice, an encouraging technique to shorten intensive care treatments and prevent postoperative complications in critically ill patients hospitalised in ICUs, is still in its infancy. Although the benefits of EM are well-known, the literature mainly focuses on the empirical results of EM, the impact of EM in the healthcare facilities and the main barriers to be addressed and relegate the integration of technology into EM practice to a secondary level. To fill this gap, this article has reviewed the relationship between the EM practice described in the literature and the use of technology. Moreover, with the smart healthcare paradigm in mind, a smart healthcare-enhanced early mobilisation scenario has been proposed by describing the main components and actors to be considered over the development of the solution.

More specifically, the motivation of this article revolves around three main cornerstones, namely Q1, Q2 and Q3, as described in Section 1.3. First, regarding Q1 (does the relevant literature on EM practice consider the use of technology?), we have observed that the relevant literature on EM practice does not consider the use nor the impact of technology. Indeed, recent surveys on the topic place little emphasis on technology and, when discussed, only machinery or technological applications are briefly detailed. Occasionally forgotten, neuromuscular electrical stimulation (NES)-based technology, robotics, mechatronics and sensors are the main technological solutions that can significantly contribute to enhance EM practice. This observation seems controversial with the maturity of many technologies already applied in other clinical daily practices. For instance, the use of NES within the EM practice is studied in [34], whereas the general use of technology is addressed in [26]. However, the latter does not mention the use of technology, such as sensors for monitoring the EM practise, nor robotics to help perform mobilisations.

Second, despite the above, we have identified a significant number of original research articles/studies on the application of technology to EM. Hence, concerning Q2 (does the literature on ICT include applications to EM?), we can state that there is certain interest in the engineering area to provide solutions to improve EM practice. Besides, it is worth noting that most of these works have been published in the last five years (five out of the eight reviewed articles have been published from 2015 onwards), thus demonstrating that the synergies between EM and technologies are gaining momentum. Notwithstanding, no proposal addresses integrating different technologies to contribute to the EM practice globally.

Furthermore, third, with regards to Q3 (how can technology help overcome barriers to EM practice?), we have proposed a novel solution to integrate existing solutions in the fields of engineering and ICT into a platform for smart healthcare-enhanced EM. This platform includes information sources (e.g., Electronic Health Records, real-time data from patient and context), sensorised monitoring of EM activities and solutions, such as NES and robotised elements, to help perform EM protocols. Our approach can help overcome the provider and institutional level barriers mentioned in Section 1.1, related to guidelines, 
management and communication between practitioners. Last but not least, our proposal can integrate solutions from the rehabilitation area, concerned with the continuity of the EM programmes in the hospital rooms or even at home, and proposals aiming at enhancing patients adherence.

All things considered, we believe that the smart healthcare paradigm can significantly contribute to improving EM and help overcome some of its barriers, especially those related to the lack of resources and management. Hence, we sustain that envisaging a smart healthcare-enhanced EM solution, working on its deployment and assessing its performance and cost/benefit would be of great interest for the medical community. Notwithstanding, the research community is only scratching the surface of the many opportunities within this field. Hence, future work will focus on the maturity of further smart-healthcare-based EM solutions from a technical perspective and addressing subsequent issues, such as interoperability, wireless channel characterisation, ethics, information security and data privacy. Latter, the implementation and validation of these solutions in real settings will be paramount to assess the benefits and suitability of technology into EM.

Author Contributions: Conceptualisation, M.F. and A.M.-B.; methodology, A.M.-B. and M.F.; investigation, M.F.; writing-original draft preparation, A.M.-B., E.B. and A.S.; writing-review and editing A.S.; visualisation, M.F., E.B. and A.M.-B.; supervision, A.M.-B. and A.S.; funding acquisition, A.S. All authors have read and agreed to the published version of the manuscript.

Funding: This work was supported by the Spanish Ministry of Science and Technology with project IoTrain RTI2018-095499-B-C32, by the Government of Catalonia with projects 2017-SGR-896 and ACTUA 2020PANDE00103, by Universitat Rovira i Virgili with project 2017PFR-URV-B2-41 and by GoodBrother COST action 19121.

Institutional Review Board Statement: Not applicable.

Informed Consent Statement: Not applicable.

Data Availability Statement: The data on the screening phase of selected works is available on http:/ / www.smarttechresearch.com/shealthEM accessed on 6 August 2021.

Conflicts of Interest: The authors declare no conflict of interest.

\section{References}

1. Rawal, G.; Yadav, S.; Kumar, R. Post-intensive Care Syndrome: An Overview. J. Transl. Intern. Med. 2017, 5, 90-92. [CrossRef]

2. Morris, P.E.; Goad, A.; Thompson, C.; Taylor, K.; Harry, B.; Passmore, L.; Ross, A.; Anderson, L.; Baker, S.; Sanchez, M.; et al. Early intensive care unit mobility therapy in the treatment of acute respiratory failure. Crit. Care Med. 2008, 36, 2238-2243. [CrossRef] [PubMed]

3. Miranda Rocha, A.R.; Martinez, B.P.; Maldaner da Silva, V.Z.; Forgiarini Junior, L.A. Early mobilization: Why, what for and how? Med. Intensiv. 2017, 41, 429-436. [CrossRef] [PubMed]

4. Bognar, K.; Chou, J.W.; McCoy, D.; Sexton Ward, A.L.; Hester, J.; Guin, P.; Jena, A.B. Financial Implications of a Hospital Early Mobility Program. Intensive Care Med. Exp. 2015, 3, A758. [CrossRef]

5. Cameron, S.; Ball, I.; Cepinskas, G.; Choong, K.; Doherty, T.J.; Ellis, C.G.; Martin, C.M.; Mele, T.S.; Sharpe, M.; Shoemaker, J.K.; et al. Early mobilization in the critical care unit: A review of adult and pediatric literature. J. Crit. Care 2015, 30, 664-672. [CrossRef]

6. Dikkema, Y.; Nieuwenhuis, M.K.; van der Schans, C.P.; Mouton, L.J. Questionnaires to Assess Facilitators and Barriers of Early Mobilization in Critically Ill Patients: Which One to Choose? A Systematic Review. Clin. Nurs. Res. 2020, 30, 442-454. [CrossRef]

7. Akinremi, A.A.; Ogwu, S.; Sanya, A.O.; Sanusi, A.A.; Osinaike, B. Early Mobilization in the ICU: A Multicenter Survey of Clinicians' Knowledge, Attitude and Practices in Resource-Limited Hospital Settings. Ann. Med. Health Sci. Res. 2020, 10, 778-784.

8. Anekwe, D.E.; Koo, K.K.Y.; de Marchie, M.; Goldberg, P.; Jayaraman, D.; Spahija, J. Interprofessional Survey of Perceived Barriers and Facilitators to Early Mobilization of Critically Ill Patients in Montreal, Canada. J. Intensive Care Med. 2017, 34, 218-226. [CrossRef]

9. Dubb, R.; Nydahl, P.; Hermes, C.; Schwabbauer, N.; Toonstra, A.; Parker, A.M.; Kaltwasser, A.; Needham, D.M. Barriers and Strategies for Early Mobilization of Patients in Intensive Care Units. Ann. Am. Thorac. Soc. 2016, 13, 724-730. [CrossRef]

10. Phelan, S.; Lin, F.; Mitchell, M.; Chaboyer, W. Implementing early mobilisation in the intensive care unit: An integrative review. Int. J. Nurs. Stud. 2018, 77, 91-105. [CrossRef]

11. Lang, J.K.; Paykel, M.S.; Haines, K.J.; Hodgson, C.L. Clinical Practice Guidelines for Early Mobilization in the ICU: A Systematic Review. Crit. Care Med. 2020, 48, e1121-e1128. [CrossRef] 
12. Eysenbach, G. What is e-health? J. Med. Internet Res. 2001, 3, e20. [CrossRef] [PubMed]

13. Ball, M.J.; Lillis, J. E-health: Transforming the physician/patient relationship. Int. J. Med. Inform. 2001, 61, 1-10. [CrossRef]

14. Istepanian, R.; Laxminarayan, S.; Pattichis, C.S. M-Health: Emerging Mobile Health Systems; Springer: Berlin/Heidelberg, Germany, 2006.

15. Cechetti, N.P.; Bellei, E.A.; Biduski, D.; Rodriguez, J.P.M.; Roman, M.K.; De Marchi, A.C.B. Developing and implementing a gamification method to improve user engagement: A case study with an $\mathrm{m}$-Health application for hypertension monitoring. Telemat. Inform. 2019, 41, 126-138. [CrossRef]

16. Batista, E.; Borras, F.; Martinez-Balleste, A. Monitoring people with MCI: Deployment in a real scenario for low-budget smartphones. In Proceedings of the 2015 6th International Conference on Information, Intelligence, Systems and Applications (IISA), Corfu, Greece, 6-8 July 2015.

17. Solanas, A.; Patsakis, C.; Conti, M.; Vlachos, I.; Ramos, V.; Falcone, F.; Postolache, O.; Pérez-Martínez, P.; Pietro, R.; Perrea, D.; et al. Smart health: A context-aware health paradigm within smart cities. IEEE Commun. Mag. 2014, 52, 74-81. [CrossRef]

18. Patsakis, C.; Papageorgiou, A.; Falcone, F.; Solanas, A. s-health as a driver towards better emergency response systems in urban environments. In Proceedings of the 2015 IEEE International Symposium on Medical Measurements and Applications (MeMeA) Proceedings, Turin, Italy, 7-9 May 2015.

19. Casino, F.; Patsakis, C.; Batista, E.; Borràs, F.; Martínez-Ballesté, A. Healthy Routes in the Smart City: A Context-Aware Mobile Recommender. IEEE Softw. 2017, 34, 42-47. [CrossRef]

20. Solanas, A.; Casino, F.; Batista, E.; Rallo, R. Trends and challenges in smart healthcare research: A journey from data to wisdom. In Proceedings of the 2017 IEEE 3rd International Forum on Research and Technologies for Society and Industry (RTSI), Modena, Italy, 11-13 September 2017.

21. vom Brocke, J.; Simons, A.; Niehaves, B.; Riemer, K.; Plattfaut, R.; Cleven, A. Reconstructing the Giant: On the Importance of Rigour in Documenting the Literature Search Process. In Proceedings of the 17th European Conference on Information Systems (ECIS), Verona, Italy, 8-10 June 2009; pp. 2206-2217.

22. Clarissa, C.; Salisbury, L.; Rodgers, S.; Kean, S. Early mobilisation in mechanically ventilated patients: A systematic integrative review of definitions and activities. J. Intensive Care 2019, 7, 3. [CrossRef] [PubMed]

23. Nweke, H.F.; Teh, Y.W.; Al-Garadi, M.A.; Alo, U.R. Deep learning algorithms for human activity recognition using mobile and wearable sensor networks: State of the art and research challenges. Expert Syst. Appl. 2018, 105, 233-261. [CrossRef]

24. Aksnes, D.W.; Langfeldt, L.; Wouters, P. Citations, Citation Indicators, and Research Quality: An Overview of Basic Concepts and Theories. SAGE Open 2019, 9. [CrossRef]

25. Mayer, K.P.; Carper, R.A.; Henson, S.C.; Clonce, E.A.; Christian, W.J.; Seif, S.M.; Pastva, A.M.; Needham, D.M.; Morris, P.E. ThreeFourths of ICU Physical Therapists Report Use of Assistive Equipment and Technology in Practice: Results of an International Survey. J. Acute Care Phys. Ther. 2020, 12, 21-30. [CrossRef]

26. Needham, D.M.; Truong, A.D.; Fan, E. Technology to enhance physical rehabilitation of critically ill patients. Crit. Care Med. 2009, 37, S436-S441. [CrossRef]

27. Wittmer, V.L.; Paro, F.M.; Duarte, H.; Capellini, V.K.; Barbalho-Moulim, M.C. Early mobilization and physical exercise in patients with COVID-19: A narrative literature review. Complement. Ther. Clin. Pract. 2021, 43, 101364. [CrossRef]

28. Goodwin, V.A.; Allan, L.; Bethel, A.; Cowley, A.; Cross, J.L.; Day, J.; Drummond, A.; Hall, A.J.; Howard, M.; Morley, N.; et al Rehabilitation to enable recovery from COVID-19: A rapid systematic review. Physiotherapy 2021, 111, 4-22. [CrossRef] [PubMed]

29. Atashzar, S.F.; Carriere, J.; Tavakoli, M. Review: How Can Intelligent Robots and Smart Mechatronic Modules Facilitate Remote Assessment, Assistance, and Rehabilitation for Isolated Adults With Neuro-Musculoskeletal Conditions? Front. Robot. AI 2021, 8, 610529. [CrossRef]

30. Zayed, Y.; Kheiri, B.; Barbarawi, M.; Chahine, A.; Rashdan, L.; Chintalapati, S.; Bachuwa, G.; Al-Sanouri, I. Effects of neuromuscular electrical stimulation in critically ill patients: A systematic review and meta-analysis of randomised controlled trials. Aust. Crit. Care 2020, 33, 203-210. [CrossRef]

31. Selves, C.; Stoquart, G.; Lejeune, T. Gait rehabilitation after stroke: Review of the evidence of predictors, clinical outcomes and timing for interventions. Acta Neurol. Belg. 2020, 120, 783-790. [CrossRef]

32. Schwab, K.E.; To, A.Q.; Chang, J.; Ronish, B.; Needham, D.M.; Martin, J.L.; Kamdar, B.B. Actigraphy to Measure Physical Activity in the Intensive Care Unit: A Systematic Review. J. Intensive Care Med. 2019, 35, 1323-1331. [CrossRef] [PubMed]

33. Newman, A.N.L.; Gravesande, J.; Rotella, S.; Wu, S.S.; Topp-Nguyen, N.; Kho, M.E.; Harris, J.E.; Fox-Robichaud, A.; Solomon, P. Physiotherapy in the neurotrauma intensive care unit: A scoping review. J. Crit. Care 2018, 48, 390-406. [CrossRef] [PubMed]

34. Greening, N.J.; Williams, J.E.A.; Hussain, S.F.; Harvey-Dunstan, T.C.; Bankart, M.J.; Chaplin, E.J.; Vincent, E.E.; Chimera, R.; Morgan, M.D.; Singh, S.J.; et al. An early rehabilitation intervention to enhance recovery during hospital admission for an exacerbation of chronic respiratory disease: randomised controlled trial. BMJ 2014, 349, g4315. [CrossRef] [PubMed]

35. Saxena, H.; Ward, K.R.; Krishnan, C.; Epureanu, B.I. Effect of Multi-Frequency Whole-Body Vibration on Muscle Activation, Metabolic Cost and Regional Tissue Oxygenation. IEEE Access 2020, 8, 140445-140455. [CrossRef]

36. Demrozi, F.; Pravadelli, G.; Tighe, P.J.; Bihorac, A.; Rashidi, P. Joint Distribution and Transitions of Pain and Activity in Critically Ill Patients. In Proceedings of the 42nd Annual International Conference of the IEEE Engineering in Medicine \& Biology Society (EMBC), Montreal, QC, Canada, 20-24 July 2020; pp. 4534-4538. 
37. Martínez-Ballesté, A.; Gimeno, P.; Mariné, A.; Batista, E.; Solanas, A. e-PEMICU: An e-Health Platform to Support Early Mobilisation in Intensive Care Units. In Proceedings of the 10th International Conference on Information, Intelligence, Systems and Applications (IISA), Patras, Greece, 15-17 July 2019; pp. 1-6.

38. Haddara, R.; Zhou, Y.; Chinchalkar, S.; Trejos, A.L. Postoperative healing patterns in elbow using electromyography: Towards the development of a wearable mechatronic elbow brace. In Proceedings of the International Conference on Rehabilitation Robotics (ICORR), London, UK, 17-20 July 2017; pp. 1395-1400.

39. Tafreshi, A.S.; Klamroth-Marganska, V.; Nussbaumer, S.; Riener, R. Real-Time Closed-Loop Control of Human Heart Rate and Blood Pressure. IEEE Trans. Biomed. Eng. 2015, 62, 1434-1442. [CrossRef]

40. Colombo, G.; Schreier, R.; Mayr, A.; Plewa, H.; Rupp, R. Novel Tilt Table with Integrated Robotic Stepping Mechanism: Design Principles and Clinical Application. In Proceedings of the 9th International Conference on Rehabilitation Robotics (ICORR), Chicago, IL, USA, 28 June-1 July 2005; pp. 227-230.

41. Tsukamoto, S.; Higashi, Y.; Nambu, M.; Sekine, M.; Nakajima, K.; Tamura, T. Visualization of transfer motion based on accelerometry data in the hemiplegic patients. In Proceedings of the Second Joint 24th Annual Conference and the Annual Fall Meeting of the Biomedical Engineering Society \& Engineering in Medicine and Biology, Houston, TX, USA, 23-26 October 2002; pp. 2457-2458.

42. Nakajima, K.; Tamura, T.; Lu, L.; Miike, H.; Kasaoka, S.; Nakashima, K.; Maekawa, T.; Togawa, T. Evaluation of bed rest using a bed temperature monitor after acute myocardial infarction. In Proceedings of the 17th International Conference of the Engineering in Medicine and Biology Society (EMBC), Montreal, QC, Canada, 20-23 September 1995; Volume 1, pp. 689-690.

43. Polastri, M.; Brini, S.; Ghetti, A.; Lama, A. Recommendations from scientific/professional societies: An essential support for physiotherapy in patients with COVID-19. Int. J. Ther. Rehabil. 2020, 27, 1-3. [CrossRef]

44. Iannaccone, S.; Castellazzi, P.; Tettamanti, A.; Houdayer, E.; Brugliera, L.; de Blasio, F.; Cimino, P.; Ripa, M.; Meloni, C.; Alemanno, F.; et al. Role of Rehabilitation Department for Adult Individuals With COVID-19: The Experience of the San Raffaele Hospital of Milan. Arch. Phys. Med. Rehabil. 2020, 101, 1656-1661. [CrossRef] [PubMed]

45. Thomas, P.; Baldwin, C.; Bissett, B.; Boden, I.; Gosselink, R.; Granger, C.L.; Hodgson, C.; Jones, A.Y.; Kho, M.E.; Moses, R.; et al. Physiotherapy management for COVID-19 in the acute hospital setting: Clinical practice recommendations. J. Physiother. 2020, 66, 73-82. [CrossRef] [PubMed]

46. Ceravolo, M.G.; Arienti, C.; de Sire, A.; Andrenelli, E.; Negrini, F.; Lazzarini, S.G.; Patrini, M.; Negrini, S.; The International Multiprofessional Steering Committee of Cochrane Rehabilitation REH-COVER Action. Rehabilitation and COVID-19: The Cochrane Rehabilitation 2020 rapid living systematic review. Eur. J. Phys. Rehabil. Med. 2020, 56, 642-651. [PubMed]

47. Stylianides, N.; Dikaiakos, M.D.; Gjermundrød, H.; Panayi, G.; Kyprianou, T. Intensive Care Window: Real-Time Monitoring and Analysis in the Intensive Care Environment. IEEE Trans. Inf. Technol. Biomed. 2011, 15, 26-32. [CrossRef] [PubMed]

48. Ortiz de Lejarazu, A.; López-Iturri, P.; Aguirre, E.; Azpilicueta, L.; Falcone, F.; Casino, F.; Solanas, A. Challenges in the implementation of context-aware scenarios within emergency rooms. In Proceedings of the 6th International Conference on Information, Intelligence, Systems and Applications (IISA), Corfu, Greece, 6-8 July 2015; pp. 1-4.

49. Casino, F.; López-Iturri, P.; Aguirre, E.; Azpilicueta, L.; Falcone, F.; Batista, E.; Solanas, A. Two-dimensional collaborative filtering approach to wireless channel characterization in medical complex scenarios. In Proceedings of the IEEE International Smart Cities Conference (ISC2), Trento, Italy, 12-15 September 2016; pp. 1-6.

50. Kang, J.J.; Dibaei, M.; Luo, G.; Yang, W.; Zheng, X. A Privacy-Preserving Data Inference Framework for Internet of Health Things Networks. In Proceedings of the IEEE 19th International Conference on Trust, Security and Privacy in Computing and Communications (TrustCom), Guangzhou, China, 29 December-1 January 2021; pp. 1209-1214.

51. Coban, C.; Tuysuz, M.F. E-Health and Privacy: Risks, Opportunities and Solutions. In Proceedings of the 4th International Conference on Computer Science and Engineering (UBMK), Samsun, Turkey, 11-15 September 2019; pp. 554-559.

52. Zhang, Y.; Zheng, D.; Deng, R.H. Security and Privacy in Smart Health: Efficient Policy-Hiding Attribute-Based Access Control. IEEE Internet Things J. 2018, 5, 2130-2145. [CrossRef]

53. Halpern, N.A. Innovative Designs for the Smart ICU. Chest 2014, 145, 646-658. [CrossRef]

54. Koller-Hodac, A.; Leonardo, D.; Walpen, S.; Felder, D. A novel robotic device for knee rehabilitation improved physical therapy through automated process. In Proceedings of the 3rd IEEE RAS \& EMBS International Conference on Biomedical Robotics and Biomechatronics, Tokyo, Japan, 26-29 September 2010; pp. 820-824.

55. Low, F.Z.; Lim, J.H.; Kapur, J.; Yeow, R.C.H. Effect of a Soft Robotic Sock Device on Lower Extremity Rehabilitation Following Stroke: A Preliminary Clinical Study With Focus on Deep Vein Thrombosis Prevention. IEEE J. Transl. Eng. Health Med. 2019, 7, 1-6. [CrossRef] [PubMed]

56. Liu, Z.; Zhao, L.; Yu, P.; Yang, T.; Li, N.; Yang, Y.; Liu, L. A Wearable Bionic Soft Exoskeleton Glove for Stroke Patients. In Proceedings of the IEEE 8th Annual International Conference on CYBER Technology in Automation, Control, and Intelligent Systems (CYBER), Tianjin, China, 19-23 July 2018; pp. 932-937.

57. Petersen, I.L.; Nowakowska, W.; Ulrich, C.; Struijk, L.N.S.A. A Novel sEMG Triggered FES-Hybrid Robotic Lower Limb Rehabilitation System for Stroke Patients. IEEE Trans. Med Robot. Bionics 2020, 2, 631-638. [CrossRef]

58. Pittaccio, S.; Garavaglia, L.; Molteni, E.; Guanziroli, E.; Zappasodi, F.; Beretta, E.; Strazzer, S.; Molteni, F.; Villa, E.; Passaretti, F. Can passive mobilization provide clinically-relevant brain stimulation? A pilot eeg and nirs study on healthy subjects. In Proceedings 
of the 35th Annual International Conference of the IEEE Engineering in Medicine and Biology Society (EMBC), Osaka, Japan, 3-7 July 2013; pp. 3547-3550.

59. Fang, Q.; Mahmoud, S.S.; Kumar, A.; Gu, X.; Fu, J. A Longitudinal Investigation of the Efficacy of Supported In-Home Post-Stroke Rehabilitation. IEEE Access 2020, 8, 138690-138700. [CrossRef]

60. Ayoade, M.; Baillie, L. A novel knee rehabilitation system for the home. In Proceedings of the SIGCHI Conference on Human Factors in Computing Systems, Toronto, ON, Canada, 26 April-1 May 2014; pp. 2521-2530.

61. Cheng, X.; Mei, X.; Hu, Y.; Fang, Y.; Wu, S.; You, F.; Kuang, S. Development of an E-Health App for Lower Limb Postoperative Rehabilitation Based on Plantar Pressure Analysis. Appl. Sci. 2018, 8, 766. [CrossRef]

62. Agyeman, M.O.; Al-Mahmood, A.; Hoxha, I. A Home Rehabilitation System Motivating Stroke Patients with Upper and/or Lower Limb Disability. In Proceedings of the 3rd International Symposium on Computer Science and Intelligent Control, Amsterdam, The Netherlands, 25-27 September 2019; pp. 1-6.

63. Jayasree-Krishnan, V.; Gamdha, D.; Goldberg, B.S.; Ghosh, S.; Raghavan, P.; Kapila, V. A Novel Task-Specific Upper-Extremity Rehabilitation System with Interactive Game-Based Interface for Stroke Patients. In Proceedings of the International Symposium on Medical Robotics (ISMR), Atlanta, GA, USA, 3-5 April 2019; pp. 1-7.

64. Steffen, D.; Muhm, M.; Christmann, C.; Bleser, G. A usability evaluation of a mobile exergame for ankle joint exercises. In Proceedings of the IEEE 6th International Conference on Serious Games and Applications for Health (SeGAH), Vienna, Austria, 16-18 May 2018; pp. 1-8.

65. Du, Z.; Sun, Y.; Su, Y.; Dong, W. A ROS/Gazebo based method in developing virtual training scene for upper limb rehabilitation. In Proceedings of the IEEE International Conference on Progress in Informatics and Computing, Shanghai, China, 16-18 May 2014; pp. 307-311.

66. Rutkowski, S.; Buekers, J.; Rutkowska, A.; Cieślik, B.; Szczegielniak, J. Monitoring Physical Activity with a Wearable Sensor in Patients with COPD during In-Hospital Pulmonary Rehabilitation Program: A Pilot Study. Sensors 2021, 21, 2742. [CrossRef]

67. Monaco, V.; Galardi, G.; Coscia, M.; Martelli, D.; Micera, S. Design and Evaluation of NEUROBike: A Neurorehabilitative Platform for Bedridden Post-Stroke Patients. IEEE Trans. Neural Syst. Rehabil. Eng. 2012, 20, 845-852. [CrossRef] [PubMed] 